\title{
Experimento de enseñanza como una aproximación metodológica a la in- vestigación en Educación Matemática
}

\section{Teaching experiments as a methodological approach to Mathematics Education Research}

\author{
Melania Bernabeu* \\ (DD) : https://orcid.org/0000-0001-7121-5795 \\ Mar Moreno ${ }^{* *}$ \\ (D) : https://orcid.org/0000-0002-6687-091X \\ Salvador Llinares ${ }^{* * *}$ \\ (D) : https://orcid.org/0000-0002-0801-316X
}

Tipo de Artículo: Informes de Investigación y ensayos inéditos

Doi: 10.17533/udea.unipluri.19.2.07

Cómo citar este artículo:

Bernabeu, M., Moreno, M., y Llinares, S. (2019). Experimento de enseñanza como una aproximación metodológica a la investigación en Educación Matemática. Uni-pluriversidad, 19(2), 103-123. doi:10.17533/udea.unipluri.19.2.07

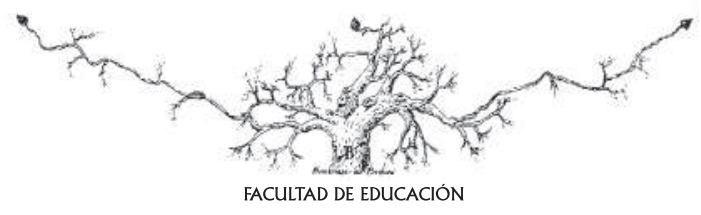

Recibido: 2019-09-23 • Aprobado: 2019-12-14

* Departamento de Innovación y Formación Didáctica, Universidad de Alicante E-mail: melania.bernabeu@ua.es

** Departamento de Innovación y Formación Didáctica, Universidad de Alicante E-mail:mmoreno@ua.es

*** Departamento de Innovación y Formación Didáctica, Universidad de Alicante E-mail: sllinares@ua.es 


\title{
Resumen
}

El experimento de enseñanza es una aproximación metodológica útil en la investigación en educación matemática para estudiar cómo los estudiantes aprenden. El objetivo de este artículo es mostrar las características de esta aproximación metodológica, a través del desarrollo de un experimento de enseñanza en tercer curso de educación primaria con estudiantes de 9 años, que está dirigido a apoyar el aprendizaje del concepto de polígono. Ejemplificamos las características de esta aproximación a la investigación en educación matemática subrayando (i) el uso de teorías sobre el aprendizaje en el diseño de las actividades, y (ii) la diversidad necesaria de fuentes de información que favorece la triangulación de las inferencias que se puedan generar. Finalmente, se apuntan posibles usos de los registros de la práctica derivados de los experimentos de enseñanza para la formación de profesores.

Palabras clave: experimento de enseñanza; educación primaria; trayectoria hipotética de aprendizaje; concepto de polígono.

\begin{abstract}
Teaching experiment is a useful methodological approach in mathematics education research to study how students learn. The objective of this article is to present the characteristics of this methodological approach, through the development of a teaching experiment in a third grade of primary education with 9-year-old students. This teaching experiment is aimed at supporting the learning about the concept of polygon. We exemplify the characteristics of this approach in mathematics education research by underlining (i) use of theories about learning in the design of activities, and (ii) diverse information sources that provide a triangulation of inferences that can emerge. Finally, possible uses of the practice records derived from teaching experiments for teacher training are noted.
\end{abstract}

Keywords: teaching experiment; primary education; hypothetical learning trajectory; concept of polygon. 


\section{LOS EXPERIMENTOS DE ENSEÑANZA COMO UNA APROXIMACIÓN A LA INVESTIGACIÓN EN EDUCACIÓN MATEMÁTICA}

Según Stylianides y Stylianides (2013), la investigación de las intervenciones en el aula (classroom-based interventions) tiene dos objetivos primordiales: (a) mejorar la práctica docente mediante aproximaciones de ingeniería didáctica para actuar sobre los problemas de la enseñanza-aprendizaje, y (b) aumentar nuestra comprensión teórica de los fenómenos que se relacionan con estos problemas. El experimento de enseñanza es una herramienta dirigida a comprender el aprendizaje y razonamiento de los estudiantes durante un corto periodo de tiempo (Steffe \& Thompson, 2000), adaptando los objetivos curriculares a los objetivos de la investigación y usando referentes teóricos para justificar el diseño de las tareas (Steffe \& Thompson, 2000).

Los experimentos de enseñanza como una aproximación metodológica en la investigación en educación matemática reúnen tres características (Stylianides \& Stylianides, 2013; p. 334):

- La investigación es conducida en clases reales, lo que inserta la investigación en el mundo de los docentes y genera un contexto para la colaboración entre profesores e investigadores, aumentando la probabilidad de que los resultados de la investigación puedan ser aplicados en el aula.

- La investigación está dirigida a problemas de aprendizaje de los estudiantes y a cómo la enseñanza puede apoyar este aprendizaje planteándose cuestiones relevantes para la práctica docente.

- Los resultados de esta aproximación a la investigación permiten desarrollar soluciones basadas en la teoría, testadas empíricamente para solventar los problemas de aprendizaje de los estudiantes mostrando qué metodología, instrumentos y acciones funcionan (o pueden funcionar) y explicando por qué funcionan.

Con estas referencias presentamos los principios que sigue esta aproximación metodológica a la investigación en educación matemática, a través de la descripción de un ejemplo de experimento de enseñanza. Dicho experimento tiene como objetivo caracterizar trayectorias de aprendizaje del concepto de polígono en estudiantes de educación primaria. Las trayectorias de aprendizaje están compuestas por descriptores de la manera de pensar de los estudiantes cada vez más sofisticadas, condicionadas por la enseñanza y los tipos de tareas propuestas (Battista, 2011; Clements \& Sarama, 2004; Seah \& Horne 2019; Simon, 1995). El análisis de las producciones de los estudiantes permite comprobar las hipótesis que justifican el diseño de las tareas. En este contexto, el investigador adopta el papel de profesor, abandonando ciertas hipótesis sobre la progresión del aprendizaje al interactuar con los estudiantes y ajustar la enseñanza a una situación concreta (Molina, Castro, Molina, \& Castro, 2011; Steffe \& Thompson, 2000). Según Cobb y Gravemeijer (2008), en un 
experimento de enseñanza se distinguen tres fases: diseño, implementación y análisis. En este artículo nos centramos en describir y caracterizar las dos primeras fases (diseño e implementación), ejemplificadas en un ex- perimento de enseñanza dirigido a potenciar el aprendizaje del concepto de polígono. Finalmente, indicamos cómo se pueden usar los resultados en los programas de formación de profesores.

\section{EXPERIMENTO DE ENSEÑANZA SOBRE EL CONCEPTO DE POLÍGONO}

La comprensión de las figuras geométricas involucra diversas acciones mentales como reconocer atributos y establecer relaciones que permitan determinar la pertenencia de una figura geométrica a una categoría (Battista, 2007; Clements, Swaminathan, Hannibal, \& Sarama, 1999; Elia \& Gagatsis, 2003; Levenson, Tirosh, \& Psamir, 2011). La comprensión del concepto de polígono es un caso particular en esta situación (Hershkowitz \& Vinner, 1983; Hershkowitz, 1989), ya que se apoya en el reconocimiento de atributos relevantes dados en la definición de polígono (figura cerrada con lados rectos y no cruzados), y atributos no-relevantes en relación con la definición (por ejemplo, concavidad-convexidad, números de lados, longitud de los lados, simetría, paralelismo), pero que son importantes para determinar clases de polígonos. Algunas investigaciones indican que los estudiantes tienen dificultades en la comprensión del concepto de polígono, lo que define un problema en la enseñanza de la geometría (Battista, 2007; Clements \& Battista, 1992; Clements et al., 1999; Gal \& Linchevski, 2010; Bernabeu \& Llinares, 2017; Bernabeu \& Moreno, 2019). En particular, los estudiantes suelen construir una limitada imagen del concepto, estructura cognitiva que se asocia con el concepto, que incluye todas las imágenes mentales $y$ propiedades y procesos asociados (Tall \& Vinner, 1981, pp. 152), tomando atributos no-relevantes como si fueran relevantes para la definición del concepto, una descripción discursiva para definir el concepto (Tall \& Vinner, 1981, pp. 152).

Una manera de dar respuesta a este problema en la enseñanza de la geometría es a través de investigaciones basadas en intervenciones en el aula, como una adaptación particular de los experimentos de enseñanza (Cobb \& Steffe, 1983; Steffe \& Thompson, 2000; Stylianides \& Stylianides, 2013). Esta aproximación metodológica intenta dar respuesta a los problemas de la enseñanza, determinando cómo apoyar el aprendizaje de los estudiantes y generando explicaciones teóricas de lo que sucede en el aula, estableciendo sinergias entre la práctica docente y la investigación (Ruthven \& Goodchild, 2008; Wiliam \& Lester, 2008).

El objetivo del presente artículo es caracterizar esta metodología de investigación en educación matemática. Para ello, se ejemplificarán los principios sobre los que se apoya, describiendo el diseño de un experimento de enseñanza dirigido al desarrollo de la comprensión del concepto de polígono en estudiantes de tercero primaria (9 años). 


\section{REFERENTES TEÓRICOS SOBRE LA COMPRENSIÓN DE LAS FIGURAS GEOMÉTRICAS}

Para diseñar el experimento de enseñanza se tuvo en cuenta el proceso de aprendizaje de la geometría desarrollado por Duval (1995). Su propuesta se apoya en la coordinación de cuatro aprehensiones: perceptiva, secuencial, discursiva y operativa. La aprehensión perceptiva se refiere a reconocer y nombrar figuras geométricas de manera global. La aprehensión secuencial es la capacidad de construir una figura o describir su construcción. La aprehensión discursiva es la habilidad de relacionar la figura con declaraciones, definiciones y atributos matemáticos que no pueden determinarse mediante la aprehensión perceptiva. Por último, la aprehensión operativa es la capacidad de modificar una figura, cambiando la posición o su orientación para resolver las tareas geométricas. Por otra parte, el estudiante necesita realizar una deconstrucción dimensio- nal (Duval, 2017) reconociendo unidades figurativas de dimensiones más pequeñas en una configuración. Por ejemplo, identificar la longitud de los lados (1D) de un triángulo (2D) para determinar su pertenencia a una determinada clase de triángulos.

\section{Diseño}

El experimento de enseñanza constó de una secuencia de enseñanza de 10 sesiones, un cuestionario contestado al inicio y final del experimento de enseñanza, y tres entrevistas realizadas a un grupo de estudiantes en tres momentos diferentes de la secuencia de enseñanza (Figura 1). En este apartado describimos las tareas usadas de cada sesión y en las entrevistas. Al finalizar cada sesión, el grupo de investigación se reunía para analizar los sucesos y realizar las modificaciones pertinentes en las sesiones siguientes.

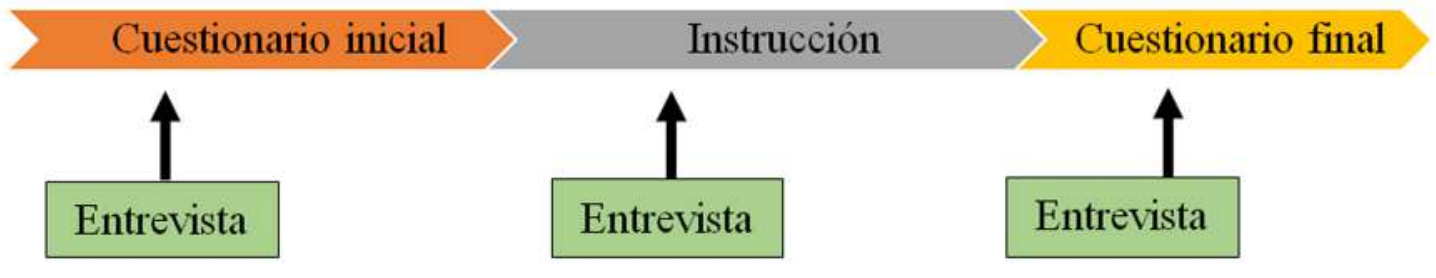

Figura 1. Secuenciación del experimento de enseñanza

Los contenidos y los procesos considerados en el experimento de enseñanza fueron la identificación de los atributos relevantes en un polígono (figura plana cerrada con lados rectos y no cruzados) y atributos que permiten determinar clases de polígonos (por ejemplo: cóncavos y convexos, número de lados, polígonos simétricos y no simétricos, tipos de triángulos según sus lados y/o sus ángulos, tipos de cuadriláteros según el paralelismo de sus lados). Además, se usaron tareas para ayudar a los estudiantes a ra- zonar con estos atributos y establecer cuándo un polígono es ejemplo de una clase. Los contenidos fueron seleccionados intencionadamente considerando el currículo, pero no necesariamente los pertenecientes al nivel educativo, ya que se introdujeron nociones relativas al paralelismo en los cuadriláteros (paralelogramos) y la clasificación de triángulos, según diferentes criterios que suelen ser introducidos en cursos posteriores para aumentar el rango de clases de polígonos que se presentarían (Tabla 1). 
Las tareas fueron diseñadas para favorecer el desarrollo de las aprehensiones y de los procesos de deconstrucción dimensional. Para ello, las tareas pedían a los estudiantes reconocer figuras geométricas y nombrarlas (aprehensión perceptiva), justificar por qué una determinada figura pertenecía a una clase de polígonos haciendo uso de las definiciones y atributos (aprehensión discursiva); transformar polígonos con determinados atributos en otros y explicar cómo era esta transformación (aprehensión secuencial y operativa).

\section{Tareas en las sesiones del experimento de enseñanza.}

Las tareas en el experimento de enseñanza exigieron a los estudiantes reconocer atributos relevantes de una figura para ser considerada un polígono, clasificar polígonos según sus atributos, dibujar polígonos con determinadas condiciones; construir polígonos con materiales manipulativos como el mecano o el geoplano, y transformar en polígono una figura que no es un polígono. En todos los casos se pidió a los estudiantes que explicaran sus respuestas. De esta manera, las tareas en las diferentes sesiones respondían a tres focos conceptuales:

- Reconocer y justificar cuando una figura es un polígono.

- Determinar la pertenencia de un polígono a una clase de polígonos (relación inclusiva), que se apoya en identificar el atributo común a conjunto de polígonos.

- Dibujar y transformar polígonos con determinados atributos.

Tabla 1. Focos de las sesiones en el experimento de enseñanza

\begin{tabular}{|c|c|c|}
\hline Sesiones & SECUENCIACIÓN & EJEMPLOS DE FIGURAS \\
\hline \multicolumn{3}{|c|}{ Cuestionario inicial } \\
\hline & $\begin{array}{l}\text { Reconocer cuando una figura es un polígono. } \\
\text { Identificar otros atributos: concavidad y con- } \\
\text { vexidad, número de lados, simetría. } \\
\text { Tipos de triángulos según sus lados, tipos de cua- } \\
\text { driláteros según el paralelismo de sus lados. }\end{array}$ & $\begin{array}{l}\text { Figura abierta con } \\
\text { un lado curvo }\end{array}$ \\
\hline \multicolumn{3}{|c|}{ Entrevista inicial } \\
\hline & $\begin{array}{l}\text { * Se consideraron los contenidos del cuestiona- } \\
\text { rio inicial, y triángulos según sus ángulos. }\end{array}$ & $\begin{array}{l}\text { Figura con un } \\
\text { lado curvo y } \\
\text { lados cruzados }\end{array}$ \\
\hline 1 & $\begin{array}{l}\text { Identificar atributos relevantes de los polígonos. } \\
\text { Identificar el número de lados. Polígonos según } \\
\text { el número de lados: triángulo, cuadrilátero, pen- } \\
\text { tágono, polígono de } 6,7 \text { y } 8 \text { lados. }\end{array}$ & Cuadrilátero \\
\hline 2 & $\begin{array}{l}\text { Diagonales. } \\
\text { Polígonos cóncavos y convexos. }\end{array}$ & Cóncavo \\
\hline 3 & $\begin{array}{l}\text { Ejes de simetría. } \\
\text { Figuras geométricas simétricas. }\end{array}$ & Eje de simetría \\
\hline
\end{tabular}




\begin{tabular}{|c|c|c|}
\hline Sesiones & SECUENCIACIÓN & EJEMPLOS DE FIGURAS \\
\hline 4 & Tipos de ángulos: agudo, recto y obtuso. & Áng \\
\hline \multicolumn{3}{|c|}{ Entrevista intermedia } \\
\hline & $\begin{array}{l}\text { Atributos relevantes de los polígonos. } \\
\text { Otros atributos de los polígonos: cóncavos y } \\
\text { convexos, número de lados, simetría. } \\
\text { Tipos de ángulos: agudo, recto y obtuso. }\end{array}$ & Pentágono cóncavo no-simétrico \\
\hline 5 & $\begin{array}{l}\text { Tipos de triángulos según la longitud de sus la- } \\
\text { dos: equilátero, isósceles y escaleno }\end{array}$ & \\
\hline 6 & $\begin{array}{l}\text { Tipos de triángulos según la amplitud de sus } \\
\text { ángulos internos: acutángulo, rectángulo y ob- } \\
\text { tusángulo }\end{array}$ & Obtusángulo \\
\hline 7 & $\begin{array}{l}\text { Clases de triángulos: triángulos según la longi- } \\
\text { tud de sus lados y según la amplitud de sus án- } \\
\text { gulos internos }\end{array}$ & Triángulo isósceles rectángulo \\
\hline 8 & $\begin{array}{l}\text { Líneas paralelas. } \\
\text { Clases de cuadriláteros según el paralelismo de } \\
\text { sus lados: paralelogramo, trapecio y trapezoide }\end{array}$ & Trapezoide \\
\hline 9 & $\begin{array}{l}\text { Clases de paralelogramos: cuadrado, rectángulo, } \\
\text { rombo y romboide }\end{array}$ & \\
\hline 10 & $\begin{array}{l}\text { Propiedades de las diagonales en los paralelo- } \\
\text { gramos: bisecan; congruentes en el cuadrado y } \\
\text { rectángulo; perpendiculares en el cuadrado y } \\
\text { rombo }\end{array}$ & \\
\hline \multicolumn{3}{|c|}{ Cuestionario final } \\
\hline & *Contenidos del cuestionario inicial. & $\nabla$ \\
\hline \multicolumn{3}{|c|}{ Entrevista final } \\
\hline & $\begin{array}{l}\text { Clases de triángulos según sus lados y/o según } \\
\text { sus ángulos. } \\
\text { Clases de cuadriláteros según el paralelismo de } \\
\text { sus lados. }\end{array}$ & ¿Es un triángulo isósceles acutángulo? \\
\hline
\end{tabular}


A continuación, se describen las tareas de las sesiones del experimento de enseñanza.

Sesión 1. En esta sesión se ejemplificaron los atributos relevantes que constituyen la definición de polígono, mostrando ejemplos de polígonos y no-polígonos. Las tareas propuestas pedían reconocer si las figuras geométricas cumplían los atributos relevantes para ser un polígono y transformar ejemplos de no-polígono en polígonos (figura abierta con lados cruzados, polígono, figura cerrada con lados curvos,...). Además, se realizaron tareas que pedían reconocer, clasificar y dibujar polígonos según el número de lados (triángulo, cuadrilátero, pentágono, polígono de 6,7 y 8 lados).

Los estudiantes contestaron dos tareas de forma individual al final de la sesión. En la primera se les pedía transformar en polígonos dos figuras que no eran polígonos y explicar la transformación realizada (Figura 2). En la segunda tarea se les pedía justificar porqué dos descripciones de figuras no eran ejemplos de polígonos.

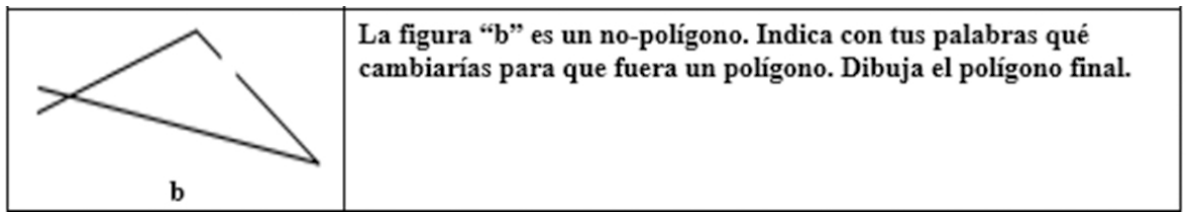

Figura 2. Apartado b de la tarea individual 1

Sesión 2. En esta sesión se ejemplificó la idea de diagonal con situaciones en diferentes tipos de polígonos. Posteriormente, los estudiantes realizaron tareas para reconocer como diagonales segmentos dibujados en rojo en las representaciones de polígonos mostrados en la pizarra digital. El significado de diagonal ayudó a introducir los polígonos cóncavos y convexos. En este experimento consideramos un polígono cóncavo como un polígono con al menos una diagonal por fuera del polígono, y polígono con- vexo como un polígono con todas las diagonales dentro del polígono. Las tareas pedían reconocer polígonos cóncavos y convexos, clasificarlos, y representarlos (Figura 3). En las tareas de esta sesión se combinaron diferentes atributos introducidos en la sesión anterior (por ejemplo, polígonos según el número de lados). Así, los estudiantes tuvieron que reconocer y representar polígonos según el número de lados y su concavidad o convexidad.
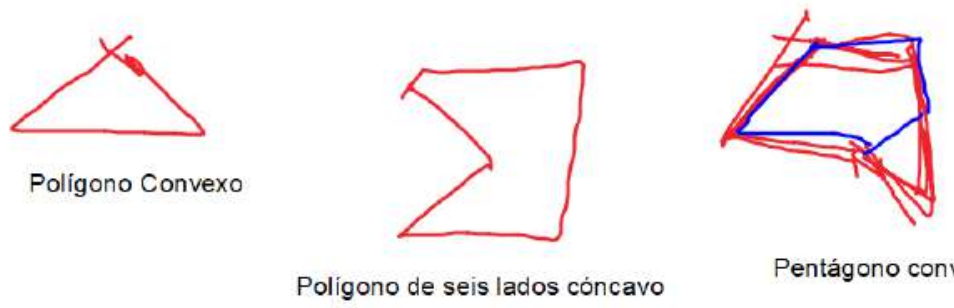

Pentágono convexo

Figura 3. Respuestas a una de las tareas de la sesión 2: Representa polígonos convexos y cóncavos (el dibujo en azul pertenece a la corrección de un estudiante al dibujo realizado por su compañero)

En las tareas individuales se les pedía transformar dos polígonos según unas condiciones dadas en relación con los atributos cóncavo y convexo (Tarea 3), y justificar que los dos enunciados correspondían con los atributos de los polígonos representados (Tarea 4). 
Sesión 3. En esta sesión se ejemplificaron figuras simétricas mostrando ejemplos y no-ejemplos. Las tareas pedían reconocer si las líneas marcadas en rojo en los polígonos eran ejes de simetría y representar ejes de simetría en polígonos dibujados en la pizarra digital. En esta sesión se usaron polígonos recortados en papel y libros de espejo para que los estudiantes comprobaran si las líneas correspondían a ejes de simetría. Con los polígonos recortados, los estudiantes doblaron por dónde creían que estaban los ejes de simetría para comprobar si coincidían las dos mitades del polígono. Con los libros de espejo, se explicó cómo usarlos para comprobar si una línea corresponde con un eje de simetría. Para ello, debían poner el espe- jo sobre la línea de manera perpendicular a la hoja donde estaba representada la figura, si lo que se veía reflejado correspondía a la mitad que había detrás del espejo, esa línea era un eje de simetría; si no se veía reflejado en el espejo lo mismo que había tras este, la línea dibujada no era un eje de simetría.

En las tareas individuales se pedía justificar si la línea representada en un polígono era un eje de simetría (Tarea 5) (Figura 4), dibujar todos los ejes de simetría de cuatro polígonos dados (Tarea 6), representar un polígono con determinadas condiciones relacionadas con los ejes de simetría, y justificar y dibujar si una diagonal puede coincidir con un eje de simetría (Tarea 7).

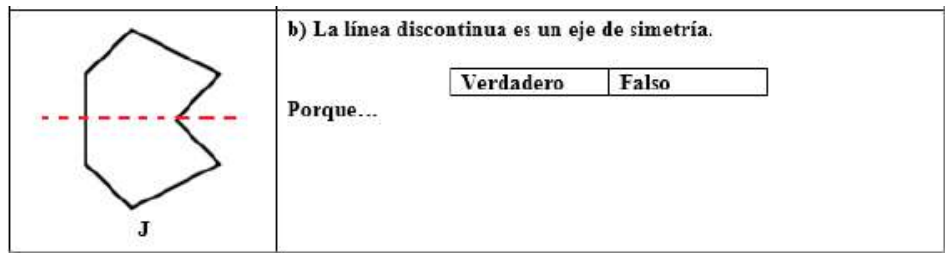

Figura 4. Apartado b de la tarea individual 5

Sesión 4. En esta sesión se ejemplificaron los diferentes tipos de ángulos según su amplitud: agudo, recto y obtuso; y se ejemplificaron estos conceptos a través de un abanico para representar ángulos agudos, rectos y obtusos. Se tomó como referencia el ángulo recto, el cual se comparó con el pico de una mesa, carpeta, pizarra, entre otros. De esta manera, el ángulo agudo es menor que el ángulo recto, el ángulo recto es como el pico de una mesa, pizarra, mesa... y el ángulo obtuso es mayor que el ángulo recto. Los estudiantes tuvieron que reconocer cómo era la amplitud de los ángulos internos de los polígonos para clasificarlos y representarlos con diferentes tipos de ángulos.

Individualmente, los estudiantes tuvieron que justificar si los ángulos internos correspondían con los marcados en los po- lígonos (Polígono "l": remarcado en líneas gruesas un ángulo interno agudo. Enunciado: Las líneas más gruesas limitan un ángulo recto) (Tarea 8). Además de representar polígonos con determinadas condiciones relacionadas con los ángulos internos de los polígonos (Tarea 9).

Sesión 5. Esta sesión se dedicó a los tipos de triángulos según la longitud de sus lados. Se usaron definiciones inclusivas: triángulo equilátero como triángulo con tres lados iguales, triángulo isósceles como triángulo con dos lados iguales, y triángulo escaleno como triángulo con tres lados diferentes. El hecho de introducir definiciones inclusivas permite considerar los triángulos equiláteros como un tipo de isósceles. Se usó el mecano para ejemplificar las explicaciones. Los estudiantes tuvieron que reconocer, 
clasificar, construir y representar triángulos con lados de diferente longitud; así como realizar transformaciones de un tipo de triángulo a otro.

En las tareas individuales se usó un geoplano isométrico para representar tres triángulos diferentes, indicar el nombre de cada triángulo y justificar su nombre (Tarea 10). La segunda tarea pedía transformar un triángulo equilátero en isósceles, y un equilátero en escaleno, explicar la transformación y dibujar los triángulos finales (Tarea 11).

Sesión 6. En esta sesión se introdujeron ejemplos de triángulos según la amplitud de sus ángulos internos: triángulo acutángulo como triángulo con los tres ángulos agudos, triángulo rectángulo como triángulo con un ángulo recto, y triángulo obtusángulo como triángulo con un ángulo obtuso. Posteriormente, se les pedía a los estudiantes reconocer, clasificar, construir y representar triángulos según la amplitud de sus ángulos internos. Con la ayuda del geoplano, en parejas, un estudiante construía uno de los triángulos representado en la pizarra digital (Figura 5) y otro compañero justificaba qué tipo de triángulo era. Además, se creó el espacio para debatir, usando el mecano, si se podía construir un triángulo rectángulo con un ángulo obtuso, un triángulo obtusángulo con un ángulo recto y finalmente, un triángulo acutángulo con un ángulo obtuso.

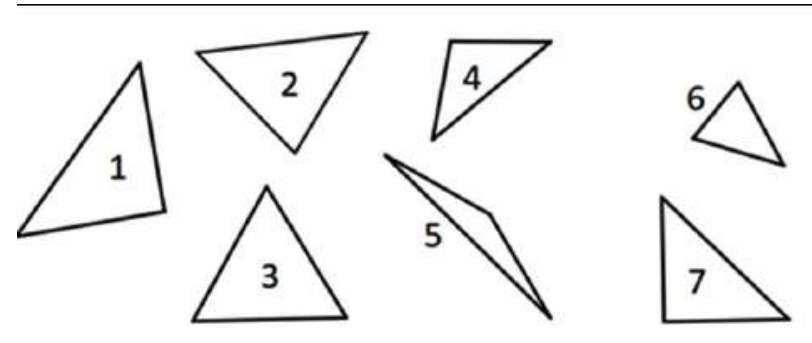

Figura 5. Ejemplos de triángulos para construir con geoplano

Las tareas individuales pedían justificar si era posible representar triángulos con ciertas condiciones en relación con la amplitud de sus ángulos (p. ej. ¿Es posible construir un triángulo rectángulo con un ángulo obtuso?) (Tarea 12), y dado un triángulo, transformarlo en otro con diferentes ángulos internos, dibujarlo y explicar la transformación (Tarea 13).

Sesión 7. Esta sesión estaba dirigida a combinar los atributos de los triángulos según sus lados y sus ángulos internos. Por ello, los estudiantes tenían que reconocer, clasificar y construir triángulos con mecano y geoplanos, según sus lados y sus ángulos. Además, transformaron triángulos para que cumplieran determinadas condiciones. Se creó el contexto para que, mediante el uso del mecano, comprobaran que no existía el triángulo equilátero rectángulo, ni el triángulo equilátero obtusángulo.

En las tareas individuales se pidió transformar dos triángulos representados en otros dos, cumpliendo determinados atributos, dibujando y explicando la transformación (Tarea 14). La segunda tarea usó la metáfora de la Máquina de Dibujar (MD) (Battista, 2012). Esta máquina solo puede hacer polígonos con determinados atributos. Los estudiantes tenían que identificar los atributos de un grupo de triángulos, hallar las semejanzas y diferencias entre ellos, y realizar una clasificación en dos grupos diferentes (p. ej., según sus lados, y explicarlo) (Tarea 15). 
Sesión 8. En esta sesión se ejemplificaron las líneas paralelas para determinar la clase de cuadrilátero. Para ello, las tareas pedían reconocer líneas paralelas y no paralelas en la pizarra digital, reconocer y clasificar cuadriláteros con y sin líneas paralelas (denominando a estos últimos como trape-

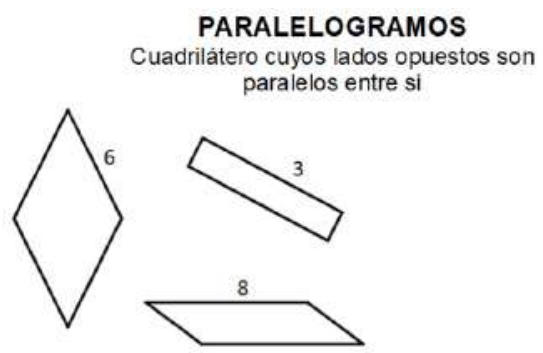

Figura 6. Clasificación de paralelogramos y trapecios realizada en la pizarra digital

Las tareas individuales consistieron en justificar si dos cuadriláteros cumplían determinados atributos (Tarea 16), clasificar un conjunto de cuadriláteros en dos grupos diferentes considerando un determinado atributo (empleando la metáfora de la MD) (Tarea 17).

Sesión 9. Las tareas de esta sesión pedían reconocer y clasificar paralelogramos. $\mathrm{Se}$ introdujeron las definiciones inclusivas de los cuatro tipos de paralelogramos: cuadrado como paralelogramo con cuatro lados iguales y todos los ángulos rectos, rectángulo como paralelogramo cuyos lados opuestos son iguales y todos los ángulos rectos, rombo como paralelogramo con cuatro lados iguales y ángulos opuestos iguales, y romboide como paralelogramo cuyos lados y ángulos opuestos son iguales.

Además, los estudiantes debían construir en parejas paralelogramos con determinadas condiciones usando el geoplano. Por ejemplo, construir un cuadrilátero con lados opuestos paralelos entre sí y cuatro ángulos iguales; o un cuadrilátero con los cuatro lados iguales y ángulos opuestos iguales. Estas tareas permitieron crear el contexto para considerar relaciones de inclusión entre los zoides). Aquí se plantearon tareas de clasificar según diferentes criterios. Por ejemplo, los trapezoides pueden ser clasificados en cóncavos y convexos, los paralelogramos en cuadriláteros con sus lados opuestos paralelos entre sí, y los trapecios en cuadriláteros con dos lados paralelos (Figura 6).

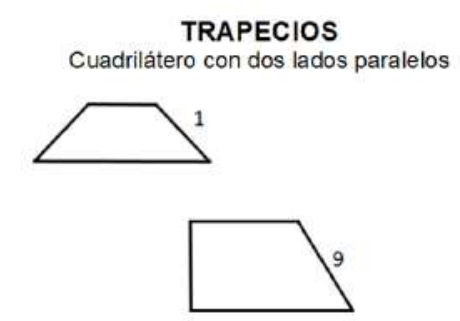

paralelogramos. Por ejemplo, considerar un cuadrado como un ejemplo de rectángulo o que un cuadrado puede ser un ejemplo de rombo (relaciones inclusivas en el conjunto de paralelogramos). El objetivo de estas tareas fue considerar un polígono como ejemplo de una clase (un tipo más específico de otro tipo más general de polígono).

En la primera tarea individual los estudiantes tenían que identificar el atributo común en un conjunto de cuadriláteros para saber cuando un polígono no pertenecía a esa clase. Los estudiantes debían explicar qué atributo define la clase y dibujar ejemplos y contraejemplos de la clase (Tarea 18). La segunda tarea consistió en transformar un determinado paralelogramo en otro según ciertas condiciones. Por ejemplo, transformar un rectángulo (cuadrilátero representado) en un cuadrado (descrito en el enunciado) y explicar qué debían cambiar para realizar dicha transformación (Tarea 19).

Sesión 10. En esta sesión se realizaron tareas con el objetivo de generar un debate centrado en identificar las propiedades de las diagonales en la clase de los paralelogramos (Figura 7). En este debate se usó el geoplano para apoyar los argumentos. En 
particular el foco de atención fue: en un paralelogramo las diagonales se bisecan, las diagonales en el cuadrado y en el rectángulo tienen la misma longitud, el rombo y el cuadrado tienen diagonales perpendiculares (si forman cuatro ángulos rectos).
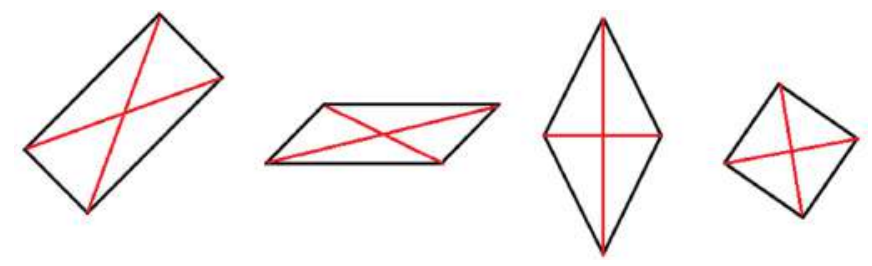

Figura 7. Representaciones de las diagonales en los paralelogramos

En la primera tarea individual se pedía dibujar un paralelogramo con diagonales perpendiculares y explicar por qué, si no fuera posible (Tarea 20). La segunda tarea consistió en explicar si las diagonales de un paralelogramo representado cumplían la descripción del enunciado presentado (por ejemplo, Paralelogramo representado: romboide. Enunciado: las diagonales miden lo mismo.) (Tarea 21).

\section{Cuestionario}

El cuestionario inicial y final constaba de las mismas tareas, lo que nos permitió comparar las respuestas de los estudiantes. El cuestionario consta de 6 tareas con un total de 12 ítems agrupados en tres focos:

(a) reconocer atributos relevantes de la definición de polígono, (b) determinar la pertenencia a una clase de polígonos:

b.1) identificar el atributo común que permite reconocer una clase de polígonos,

b.2) determinar si un polígono pertenece a la clase,

(c) dibujar polígonos con determinados atributos.

Describimos a continuación los ítems en los diferentes focos

(a) Reconocer atributos relevantes de la definición de polígono, tarea 1 (ítems $1 \mathrm{a}, 1 \mathrm{~b}, 1 \mathrm{c}, 1 \mathrm{~d}$ y $1 \mathrm{e})$

En el ítem 1a, los estudiantes tienen que reconocer en un conjunto de 15 figuras geométricas planas ( 8 polígonos y 7 no-polígonos), las figuras que son polígonos (figuras planas cerradas con lados rectos y no cruzados) (Figura 8).

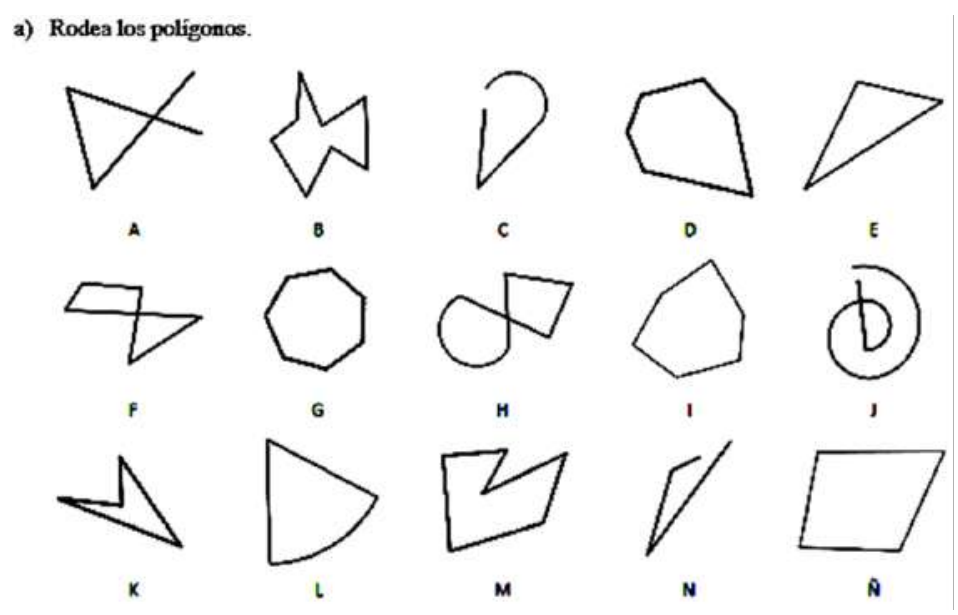

Figura 8. Ítem 1a del cuestionario 
En los ítems 1b, 1c, 1d y 1e, los estudiantes tienen que identificar los atributos que faltan en los no-polígonos para transformarlos en polígonos, explicar qué se tiene que cambiar para transformarlo y dibujar$l o$. Los no-polígonos son: una figura abierta con lados curvos y cruzados (1b), una figura abierta con un lado curvo (1c) (Figura 9), una figura cerrada con lados cruzados y un lado curvo (1d), y una figura abierta con lados cruzados (1e).

c) La figura "P" no es un poligono. Indica con tus palabras qué cambiarías para que fuera un poligono. Dibújalo.

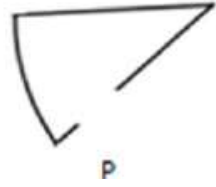

b) Determinar la pertenencia a una clase de polígonos:

b.1) Determinar la pertenencia a una clase. Identificar el atributo común en un conjunto de polígonos, tarea 2, 3 y 4 (ítems 2, 3 y 4a).

En las tareas 2, 3 y 4 se presentan dos conjuntos de polígonos, los que la Maquina de Dibujar puede (ejemplo) y no puede hacer (no-ejemplo). En uno de los grupos las figuras comparten un único atributo -el que define la clase- - y que las figuras del otro grupo no cumplen. Los estudiantes deben reconocer cuál es el atributo común, y dibujar un ejemplo de la misma clase y otra figura que no pertenezca a la clase (no-ejemplo). En la tarea 2 se presenta un conjunto de polígonos cóncavos y un conjunto de polígonos convexos (Figura 10), en la tarea 3 se presenta un conjunto de polígonos con seis lados y otro con un número de lados diferente a seis, y en la tarea 4, en el ítem $4 \mathrm{a}$, se presenta un grupo de polígonos simétricos y otro grupo con polígonos no simétricos.

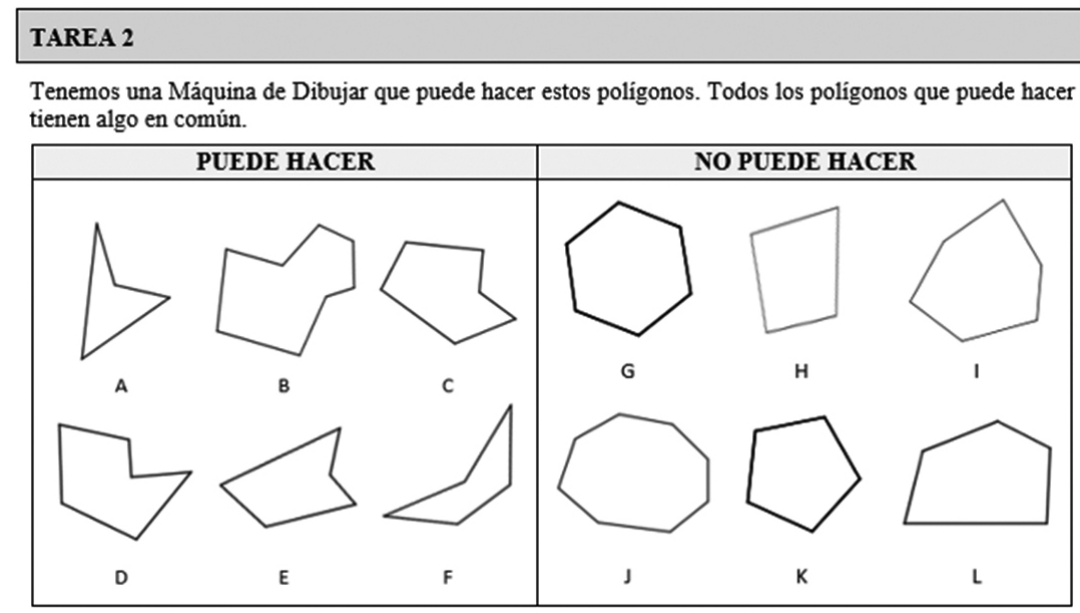

Figura 10. Tarea 2 del cuestionario: polígonos cóncavos y convexos

b.2) Determinar la pertenencia de un polígono a una clase. Clasificación de polígonos, tareas $4,5 \mathrm{y}$ 6 (ítems $4 b, 5$ y $6 b$ )
Los estudiantes tienen que identificar el atributo común del conjunto de polígonos, representados mediante dibujos o una descripción verbal, y determinar si un polígono 
pertenece o no a esta clase. Para este tipo de tareas, se considera que se clasifican los polígonos cuando los estudiantes realizan clasificaciones inclusivas. En el ítem $4 b$ se considera el rombo - figura con dos ejes de simetría- como un ejemplo de polígono con un eje de simetría (Figura 11). En la tarea 5 se considera un triángulo equilátero como un ejemplo de triángulo isósceles. Por último, el ítem $6 \mathrm{~b}$ consiste en razonar si un paralelogramo — cuadrilátero con los lados opuestos paralelos entre sí- es un ejemplo de un trapecio, - cuadrilátero con dos lados paralelos-.

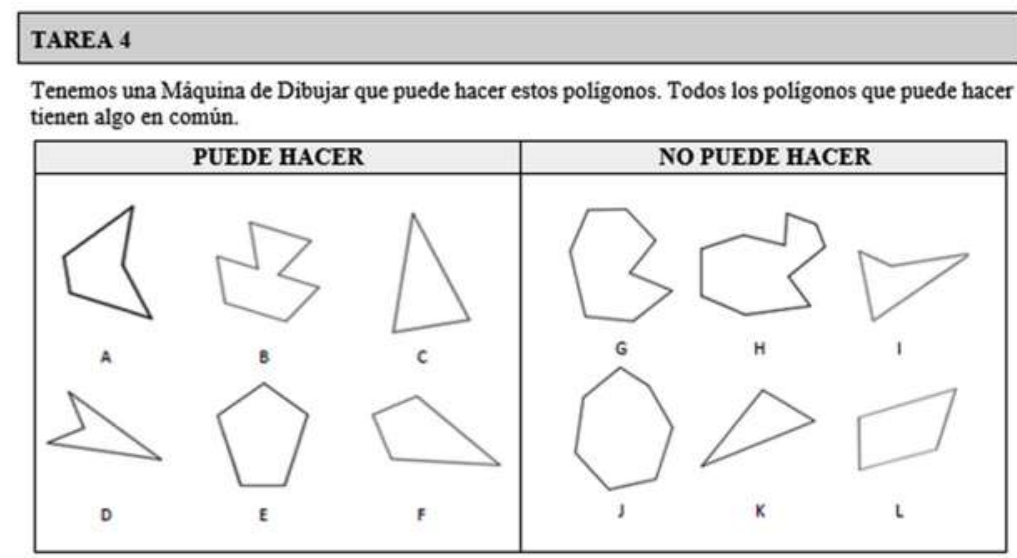

b) ¿Puede la Mâquina de Dibujar hacer la figura " $\mathrm{M}$ "? ¿ Por qué?

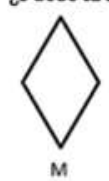

Figura 11. Ítem 4b: clasificar un rombo como un polígono con un eje de simetría

(c) Dibujar polígonos con determinados atributos, tarea 6 (ítem 6a)

El ítem 6a consiste en dibujar tres cuadriláteros con dos lados paralelos y tres cuadriláteros que no cumplan estas condiciones
(Figura 12). Para dibujar un polígono con determinadas condiciones, el estudiante debe identificar los atributos del enunciado para asociarlos con las definiciones para realizar un dibujo

\begin{tabular}{|c|c|}
\hline TAREA 6 & \\
\hline Tenemos una Máquina de Dibujar que puede ha & \\
\hline Cuadriláteros (poligonos de & ro lados) con dos lados paralelos. \\
\hline a) Dibuja tres cuadriláteros que si pueda hacer & cuadriláteros que no pueda hacer. \\
\hline $\begin{array}{c}\text { PUEDE HACER } \\
\text { (Cuadriláteros con dos lados paralelos) }\end{array}$ & NO PUEDE HACER \\
\hline 1. & 1 \\
\hline 2 & 2 \\
\hline 3 & 3 \\
\hline
\end{tabular}

Figura 12. Ítem 6a: dibujar polígonos con determinadas condiciones 


\section{Entrevistas}

Las entrevistas se realizaron en tres momentos del experimento de enseñanza: antes, durante y después de la instrucción. Las entrevistas permiten disponer de evidencias de cambios en el discurso a lo largo de la secuencia de enseñanza. Las tareas de las entrevistas son similares a las realizadas en las sesiones.

En la entrevista inicial se pedía a los estudiantes que explicaran sus respuestas al cuestionario inicial. Además, durante la entrevista se introdujeron nuevas cuestiones similares a las del cuestionario para comprobar in situ cómo los estudiantes razonaban, lo que permitía al investigador corroborar las inferencias realizadas en las respuestas al cuestionario. Las tareas de la entrevista fueron:

- La tarea 1, transformar un no-polígono en un polígono y, dibujar un no-polígono y transformarlo en un polígono. En ambos casos se debe argumentar la transformación y dibujar el nuevo polígono.

- La tarea 2, identificar el atributo común de un conjunto de polígonos. A los estudiantes se les da la consigna de que hay una Máquina de Dibujar (MD) que puede dibujar unos polígonos (cóncavos) pero no puede dibujar otros (convexos). Se pide identificar el atributo común del conjunto de polígonos que la MD podía dibujar y, dibujar un ejemplo y un contraejemplo (un polígono que la $\mathrm{MD}$ no puede dibujar).

- La tarea 3, clasificar un conjunto de triángulos en dos grupos identificando el atributo que caracteriza cada grupo y, dibujar un ejemplo de cada uno de los grupos identificados.

- La tarea 4, identificar el atributo común de un grupo de cuadriláteros que lo diferencia de otro grupo. Un grupo de cuadriláteros tiene dos lados paralelos y el otro son cuadriláteros sin lados paralelos. Se debe explicar la respuesta e identificar las similitudes entre un cuadrado $\mathrm{y}$ un rombo, y entre un cuadrado y un rectángulo.

En la entrevista intermedia se incluyeron los contenidos introducidos en las sesiones previas. El objetivo fue identificar cambios en el razonamiento de los estudiantes. Para resolver las tareas de la entrevista, los estudiantes tuvieron a su disposición materiales manipulativos como el mecano y el geoplano para hacer pruebas y razonar con los modelos, si era necesario. Los atributos en las tareas de la entrevista intermedia fueron: concavidad-convexidad, número de lados, diagonales de los polígonos, ejes de simetría, simetría de los polígonos y ángulos internos de los polígonos. Las tareas de la entrevista fueron:

- La tarea 1, reconocer los atributos que se deben transformar en un no-polígono para convertirse en polígono y dibujar el polígono resultante. Determinar la pertenencia de polígono a una clase de polígonos.

- La tarea 2, determinar la pertenencia de un polígono (pentágono cóncavo) a una clase de polígonos (cuadrilátero convexo), dada verbalmente.

- La tarea 3, dibujar polígonos con determinadas características y sus diagonales. 
Además, se incluyen preguntas sobre la caracterización de polígono cóncavo y convexo, el número de lados y reconocimiento de figuras simétricas.

- La tarea 4, identificar el atributo común de un conjunto de polígonos (ejes de simetría), a través de la metáfora de la MD, mostrando ejemplos de polígonos que la MD puede dibujar y ejemplos que no puede dibujar. Dibujar un ejemplo que cumpla el atributo identificado y un contraejemplo. Establecer relaciones entre los atributos de un polígono representado (hexágono regular con 6 ejes de simetría) y el atributo común del conjunto de polígonos que la MD puede dibujar (polígonos con dos ejes de simetría).

- La tarea 5, identificar ángulos internos en la representación de un polígono y nombrarlo.

- La tarea 6, representar dos polígonos, con condiciones dadas, que combinaban diferentes atributos.

La entrevista final se centró en los contenidos introducidos desde la entrevista intermedia hasta el final de la secuencia de enseñanza. Las tareas de la entrevista se centraban en caracterizar los triángulos atendiendo a sus lados y a sus ángulos y caracterizar los cuadriláteros atendiendo al paralelismo de sus lados. Al igual que en la entrevista intermedia, los estudiantes tenían a su disposición el mecano y el geoplano para construir modelos y explicar sus respuestas. Las tareas fueron:

- La tarea 1, determinar la pertenencia de un triángulo dado gráficamente (triángulo escaleno) como un ejemplo o no de una clase de triángulo, proporcionada verbalmente (triángulo equilátero). En el caso de no pertenecer el ejemplo de triángulo a la clase determinada, realizar las transformaciones necesarias para que pudiera pertenecer, justificando la transformación y dibujando el triángulo resultante.

- La tarea 2, determinar la pertenencia de un triángulo dado gráficamente (triángulo escaleno obtusángulo) como un ejemplo o no de una clase de triángulo, proporcionada verbalmente (triángulo isósceles acutángulo).

- La tarea 3, construir un triángulo isósceles obtusángulo con el mecano o con el geoplano y dibujarlo, explicando la respuesta. Además, se incluye una pregunta para razonar sobre el hecho de si un triángulo equilátero puede ser obtusángulo.

- La tarea 4, identificar el atributo común en un conjunto de cuadriláteros. Se usa la metáfora de la MD que permite dibujar paralelogramos con lados iguales y ángulos opuestos iguales (rombos y cuadrados), pero no puede hacer cuadriláteros que no cumplan estos atributos. Esta tarea exige reconocer los atributos comunes en un grupo, justificar el razonamiento empleado y dibujar un ejemplo y un contraejemplo. Además, se pregunta si una cometa (trapezoide convexo) cumple o no el atributo de la clase de figuras (Figura 13).

- La tarea 5, dibujar dos paralelogramos con ciertas condiciones relacionadas con las propiedades de sus diagonales. 


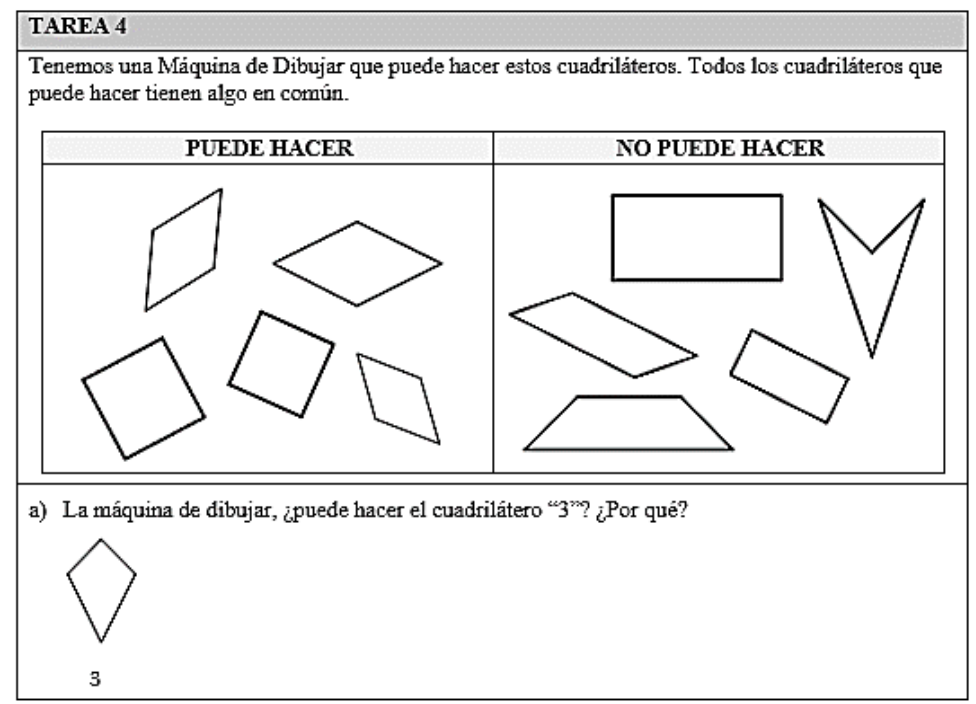

Figura 13. Tarea 4 del cuestionario de la entrevista final

\section{Implementación}

Las 10 sesiones se desarrollaron en cinco semanas ( 2 sesiones por semana) con el curso académico 2017/2018. Los estudiantes respondieron al miso cuestionario al inicio y después de las 10 sesiones. Además, un grupo de nueve estudiantes contestaron a tres entrevistas individuales en diferentes momentos del experimento de enseñanza. Estos nueve estudiantes fueron elegidos según las respuestas dadas al cuestionario inicial, con el objetivo de aportar información sobre las posibles trayectorias de aprendizaje generadas.

La realización de 2 sesiones por semana posibilitaba las reuniones del grupo de investigación para analizar lo sucedido y rediseñar las siguientes sesiones dependiendo de cómo los estudiantes resolvían las tareas. Cada sesión tenía una duración de 50 minutos en la que los estudiantes trabajaban en gran grupo, debatiendo sus respuestas y resolviendo individualmente dos o tres tareas similares a las realizadas durante la sesión. Todas las sesiones fueron videograbadas. Los datos que generó este experimento de enseñanza fueron: las respuestas del cues- tionario inicial y final, la transcripción de las tres entrevistas, los vídeos y transcripciones de las diez sesiones, y las respuestas individuales a las tareas del final de cada sesión.

Antes de empezar el experimento de enseñanza, los estudiantes contestaron al cuestionario inicial y se les explicó qué iban a hacer en las siguientes semanas. En cada sesión se recordaban los contenidos de las sesiones anteriores y se presentaban los nuevos en la pizarra digital, así como las tareas que los estudiantes debían resolver en grupo y presentar también en la pizarra digital. Los estudiantes debían explicar sus respuestas y responder a las cuestiones de sus compañeros. La investigadora-profesora actuó de mediadora, seleccionando los estudiantes que debían presentar la resolución de las tareas, teniendo en cuenta las características de sus respuestas, para que todos los estudiantes participaran. Al finalizar las diez sesiones, los estudiantes volvieron a contestar el cuestionario inicial.

El objetivo de realizar tres entrevistas en diferentes momentos fue obtener información sobre características de posibles trayectorias de aprendizaje que se podían estar 
generando. El criterio de selección de los estudiantes fue: tres estudiantes que realizaran incorrectamente casi todas las tareas del cuestionario inicial, tres que realizaran correctamente las tareas relacionadas con los atributos relevantes que determinan la definición de polígono y realizaran incorrectamente las tareas sobre los tipos de polígonos según sus atributos (cóncavos y convexos, número de lados, polígonos simétricos y no simétricos, tipos de triángulos según sus lados y tipos de cuadriláteros según el paralelismo de sus lados), y tres estudiantes que contestaran correctamente a la mayoría de las tareas en el cuestionario inicial.

La implementación de las diferentes partes del experimento de enseñanza permitió desarrollar ciclos de diseño-implementación-análisis para reajustar las decisiones iniciales.

\section{Consideraciones finales}

El objetivo de este trabajo es ejemplificar la aproximación metodológica a la investigación en educación matemática basada en el diseño de experimentos de enseñanza (classroom-based interventions) (Stylianides \& Stylianides, 2013). Se ha usado un ejemplo de experimento de enseñanza dirigido a apoyar el desarrollo de la comprensión del concepto de polígono en estudiantes de tercero primaria (9 años). Se reseña:

(i) el papel que desempeña la teoría en el diseño de las actividades, y

(ii) la necesaria diversidad de fuentes de información que permitan generar contextos para la triangulación de las inferencias que se puedan generar.

Esta metodología de investigación permite generar información, a través del análisis de los vídeos, de las respuestas las tareas individuales y de las respuestas a los cuestio- narios iniciales y finales, sobre la manera en la que las aprehensiones y la deconstrucción dimensional pueden ayudarnos a comprender el aprendizaje de los contenidos geométricos. En particular, la descripción del experimento de enseñanza y la identificación de las decisiones metodológicas ejemplifican un tipo de investigación en educación matemática dirigida a los problemas de aprendizaje de los estudiantes y cómo la enseñanza puede mejorar este aprendizaje.

La puesta en práctica de esta metodología es compleja pero resulta útil, ya que sistematiza la investigación que se realiza sobre enseñanza y aprendizaje de las matemáticas. Asimismo, al aproximarnos a la práctica de aula, los datos proporcionan una descripción más real del proceso de aprendizaje de los estudiantes, aumentando el poder explicativo de las inferencias realizadas. El control sobre las diferentes etapas del experimento de enseñanza permite a los investigadores analizar de forma continua las intervenciones, rediseñar y adaptar las tareas y la instrucción.

La aproximación metodológica a la investigación en educación matemática que usa experimentos de enseñanza puede adoptar diferentes niveles de detalle que permiten tener un rango amplio de intervenciones. Estas intervenciones pueden ir desde la aproximación clínica, como se caracterizan en las aproximaciones constructivistas, a intervenciones globales en un aula (Steffe \& Thompson, 2000). Esta diversidad de la aproximación metodológica hace necesario clarificar las características de los experimentos de enseñanza, lo que implica considerar diferentes lentes a través de las cuales ver las investigaciones (Schoenfeld, 2013).

Un valor añadido que podemos derivar de este tipo de aproximación metodológica 
en la investigación en educación matemática es la posibilidad de generar registros de la práctica, tales como videoclips del trabajo en grupo de los estudiantes o sobre las discusiones en gran grupo, respuestas escritas de los estudiantes con diferente nivel de sofisticación que ayudan a ejemplificar ideas teóricas, que pueden ser usados en la formación de profesores (Buchbinder \& Kuntze, 2018; Sánchez-matamoros, Moreno, Perez-Tyteca $\&$ Callejo, 2018). Los experimentos de enseñanza pueden aportar diferentes tipos de representaciones de la práctica de enseñar matemáticas que ejemplifican conocimiento generado por las investigaciones y que los formadores consideran relevante para ser introducido como contenido en los programas de formación. En este contexto, la idea de descomposición, representación y aproximación a la práctica subraya la relevancia que actualmente están tomando las pedagogías de formación de profesores que se apoyan en el uso de los registros de la práctica para apoyar el aprendizaje de los profesores (Sztajn, Dick, Alnizami, Heck, \& Malzahn, 2020).

\section{Agradecimientos}

Esta investigación ha sido financiada por Prometeo/2017/135 de la Generalitat Valenciana (España), con el apoyo de la Universidad de Alicante (FPU2017-014).

\section{REFERENCIAS BIBLIOGRÁFICAS}

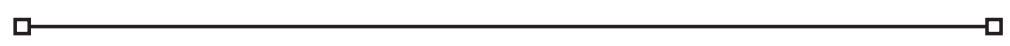

Battista, M. T. (2007). The Development of Geometric and Spatial Thinking. In, F. K. Lester (ed). Second Handbook of Research on Mathematics Teaching and Learning (pp. 843- 908). Charlotte, NC: NCTM-IAP.

Battista, M. T. (2011). Conceptualizations and issues related to learning progressions, learning trajectories, and levels of sophistication. The Mathematics Enthusiast, 8(3), 507-570.

Battista, M. T. (2012). Cognition-Based Assessment \& teaching of geometric Shapes: Building on Students' Reasoning. EEUU: Heinemann.

Bernabeu, M., \& Llinares, S. (2017). Comprensión de las figuras geométricas en niños de 6-9 años. Educación matemática, 29(2), 9-35.

Bernabeu, M., \& Moreno, M. (2019). Comprensión de los tipos de triángulos apoyados en el uso del mecano. Uno: Revista de didáctica de las matemáticas, (85), 60-65.

Buchbinder, O., \& Kuntze, S. (2018). Mathematics Teachers Engaging with Representations of Practice. A Dynamically Evolving Field. London: Springer

Clements, D. H., \& Battista, M. T. (1992). Geometry and spatial reasoning. In D. A. Grouws (Ed.), Handbook of research on mathematics teaching and learning (pp. 420-464). New York: Macmillan. 
Clements, D. H., \& Sarama, J. (2004). Learning trajectories in mathematics education. Mathematical Thinking and Learning, 6(2), 81-89.

Clements, D. H., Swaminathan, S., Hannibal, M. A. Z., \& Sarama, J. (1999). Young children's concepts of shape. Journal for Research in Mathematics Education, 30, 192-212.

Cobb, P., \& Gravemeijer, K. (2008). Experimenting to support and understand learning processes. En A.E. Kelly, R.A. Lesh, \& J.Y. Baek. (eds.). Handbook of design research methods in education. Innovations in Science, Technology, Engineering and Mathematics Learning and Teaching, pp. 68-95. Mahwah, NJ: Lawrence Erlbaum Associates.

Cobb, P., \& Steffe, L. P. (2010). The constructivist researcher as teacher and model builder. In $A$ journey in mathematics education research (pp. 19-30). Springer, Dordrecht.

Duval, R. (1995). Geometrical Pictures: Kinds of representation and specific processes. In R. Sutherland \& J. Mason (Eds.), Exploiting mental imagery with computers in mathematical education (pp. 142- 157). Berlin: Springer.

Duval, R. (2017). Understanding the mathematical way of thinking - The registers of semiotic representations. London: Springer.

Elia, I., \& Gagatsis, A. (2003). Young children's understanding of geometric shapes: The role of geometric models. European Early Childhood Education Research Journal, 11(2), 43-61.

Gal, H., \& Linchevski, L. (2010). To see or not to see: analyzing difficulties in geometry from the perspective of visual perception. Educational studies in mathematics, 74(2), 163-183.

Hershkowitz, R. (1989). Visualization in geometry - two sides of the coin. Focus on Learning Problems in Mathematics, 11(1), 61-76.

Hershkowitz, R., \& Vinner, S. (1983). The role of critical and non-critical attributes in the concept image of geometrical concepts. In Proceedings of the 7th PME International Conference (pp. 223-228).

Levenson, S., Tirosh, D., \& Tsamir, P. (2011). Preschool Geometry. Theory, Research and Practical Perspectives. Rotterdam: Sense Publishers.

Molina, M., Castro, E., Molina, J. L., \& Castro, E. (2011). Un acercamiento a la investigación de diseño a través de los experimentos de enseñanza. Enseñanza de las ciencias: revista de investigación y experiencias didácticas, 29(1), 75-88.

Ruthven, K., \& Goodchild, S. (2008). Linking researching and teaching: Towards synergy of scholarly and craft knowledge. In L. D. English (Ed.), Handbook of international research in mathematics education (2nd ed., pp. 561-588). New York, NY: Routledge.

Sánchez-matamoros, G., Moreno, M., Pérez-Tyteca, P., \& Callejo, M.L. (2018). Trayectorias de aprendizaje de La longitud y su medida como instrumento conceptual usado por futuros maestros de Educación Infantil. Revista Latinoamericana de Investigación en Matemática Educativa, 21(2), 203-228. 
Schoenfeld, A. (2013). On forests, trees, elephants, and classrooms: a brief for the study of learning ecologies. ZDM. Mathematics Education, 45(3), 491-495.

Seah, R., \& Horne, M. (2019). The construction and validation of a geometric reasoning test item to support the development of learning progression. Mathematics Education Research Journal, $1-22$.

Simon, M. A. (1995). Reconstructing mathematics pedagogy from a constructivist perspective. Journal for Research in Mathematics Education, 26(2), 114-145.

Steffe, L. P., \& Thompson, P. W. (2000). Teaching experiment methodology: Underlying principles and essential elements. Handbook of research design in mathematics and science education, 267306.

Stylianides, A. \& Stylianides, G. (2013). Seeking research-grounded solutions to problems of practice: classroom-based interventions in mathematics education. ZDM. Mathematics Education, $45(3), 333-341$.

Sztajn, P., Dick, L., Alnizami, R., Heck, D., \& Malzahn, K. (2020). Controlled implementations. Teaching Practice to Practicing mathematics Teachers. In S. Llinares, y O. Chapman (eds.), International Handbook of Mathematics Teacher Education. Volume 2: Tools and Processes in Mathematics Teacher Education, Second Edition, (p. 285-310). Leiden, The Netherland: Brill.

Tall, D., \& Vinner, S. (1981). Concept image and concept definition in mathematics with particular reference to limits and continuity. Educational Studies in Mathematics, 12(2), 151-169.

Wiliam, D., \& Lester, F. K. (2008). On the purpose of mathematics education research: Making productive contributions to policy and practice. In L. D. English (Ed.), Handbook of international research in mathematics education (2nd ed., pp. 32-48). New York, NY: Routledge. 